\title{
Effects of Different Attaching Positions of Human Body on Wearable Tag Antenna
}

\author{
Shuo $\mathrm{CHEN}^{1}$, Song $\mathrm{LI}^{2,{ }^{*}}$, Hong-zhe LIU, Fan LU and Jian MA ${ }^{3}$ \\ ${ }^{123}$ School of Logistics Engineering, Wuhan University of Technology, Wuhan 430063, \\ China
}

Keywords: Wearable tag, Impedance, Gain, Read range, UHF RFID.

\begin{abstract}
In this paper, a research on radio frequency identification (RFID) tag for body wearable is presented. Performances of tag and antenna are mainly focused on with the tag patched on human body. Results are available from simulation and measurement to reflect the change of wearable tag performance. A human model with specific posture was set to allow the tag attaching on body in simulation. Impedances and gains were obtained to evaluate the change of tag antenna performance with the tag on five different body positions. In actual measurement, read ranges of tag on different positions of body were measured. Ideal read distances of the tag were calculated using Friis free-space formula with the simulation results to compare with the measured ones. The results demonstrate the performance of tag antenna can be affected by bodily tissue absorbing and antenna bending and posture shielding. It is confirmed the antenna is suitable for UHF RFID tags in passive RFID applications.
\end{abstract}

\section{Introduction}

With the developing of RFID technology, researches on body-centric wireless system have been hot in recent years [1-4]. As an important application of RFID, Body-centric wireless systems provide several new and important features for applications in wireless sensor systems, health care, real-time monitoring of human vital signs, and neural activity [5]. Wearable antennas have an enormous potential in future welfare, healthcare, and childcare applications [5, 6]. In order to improve the antenna, wearable antenna needs to be designed in small size, large enough wideband. It should be comfortable to wear and inexpensive to widespread use [5]. Moreover, high-quality wearable antenna should be designed and optimized in bad environment. Recently, most researches on the effects of bending and on-body condition are separated. It is incomplete to research the factors isolated since the uncertain effect ratio of the two factors on wearable tags. Thus, the effect of bending and human tissue on the wearable tags should be researched with the two factors occurring synchronously.

\section{The Wearable Tag Antenna and Human Posture}

Passive tag gets activated with RF energy from RFID reader and it extracts information from the tag[7].As an important application, the RFID tag is composed of two entities: the tag antenna and the RFID tag chip. In this paper, the tag works at $902-928 \mathrm{MHz}$ in North and South America. The proposed antenna geometry originally presented in paper [8-10], which works at the center frequency of the European UHF RFID band. Parameters of the antenna in this paper were changed with ANSYS HFSS to make the antenna work at the RFID UHF bands of 902-928 MHz [11]. Fig.1 presents the tag antenna geometry. In CST, impedance of the tag antenna is $(12.8+\mathrm{j} 197.7) \Omega$ and gain is 
$2.05 \mathrm{~dB}$. The size of antenna is small enough to attach to parts of human body such as the wrist.

Advantages of screen printing include low cost of equipment, miniaturization and the faster and easier production of complex structures [12]. Polyethylene terephthalate material is widely used in flexible antenna research. The wearable tag antenna was manufactured by screen printing silver paste on PET substrates. With PET( $=2.6$ and tan 0.002) substrate, one-layer conductive silver paste is printed for easily integrated with PET compared with copper tape. The RFID is a passive smart chip with operating frequency band of $840 \mathrm{MHz} \sim 960 \mathrm{MHz}$, and the input impedance of the chip is $(12.7-\mathrm{j} 199) \Omega$ at $915 \mathrm{MHz}$.
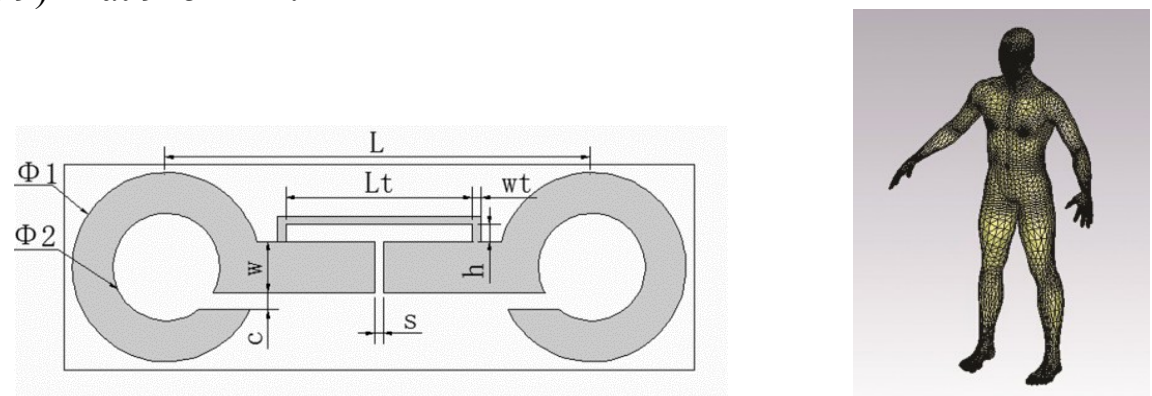

Fig.1. The tag antenna geometry, dimensions in mm. Fig.2. Human model L 50.2mm, Lt 22mm, w 6mm, $w_{t} 1 \mathrm{~mm}, \mathrm{c} 2 \mathrm{~mm}, \mathrm{~s} 1 \mathrm{~mm}, \Phi 122.2 \mathrm{~mm}, \Phi 212.6 \mathrm{~mm}$

\section{Simulation}

Antenna can be attached to model of the human body in electromagnetic software to simulate the situation of tag antenna on body and research the influence of antenna. To make the simulation accurate, accuracy of the model needs to be high enough.Presented in Fig.2, model of human body is $180 \mathrm{~cm}$ high and much similar to human in appearance. The model was set by 2 nd order model with human's dielectric constant.

In practice, wearable things are bent to some extent when they are attached on some positions of human body, such as the wrist. In this paper, five positions of body (chest, back, up arm, wrist and thigh) were selected to attach wearable tags for their wide range of practical applications in this paper. Tag antenna attached on three of the positions (up arm, wrist and thigh) was bent in simulation in order to accord with reality. Another key factor of tag is the distance between human body and the tag as mentioned before. In simulation, distances between geometrical center of tag antennas and human model are about $10 \mathrm{~mm}$ in five situations.

Impedance determines the matching degree between tag antenna and tag chip. Generally, a tag antenna with dipole structure has a large variation in input reactance, especially near materials with high conductivity. The variation of input impedance leads to a large impedance mismatch and hinder the performance of the tag. Fig.3(A,B) is the simulation results of antenna impedance under five situations. All of the five situations have values of resistance bigger than $12.7 \Omega$, and values of the reactance are far from $\mathrm{j} 200 \Omega$. Antenna geometry includes a T-match structure which is used to adjust the impedance. Bending and attaching on the model affected the structure and led to the phenomenon of mismatch. At the frequency of $915 \mathrm{MHz}$, the matching coefficients of all five tag antennas and chips were changed to $0.11-0.23$ by calculation. This effect could be avoided by optimizing the T-match structure. 

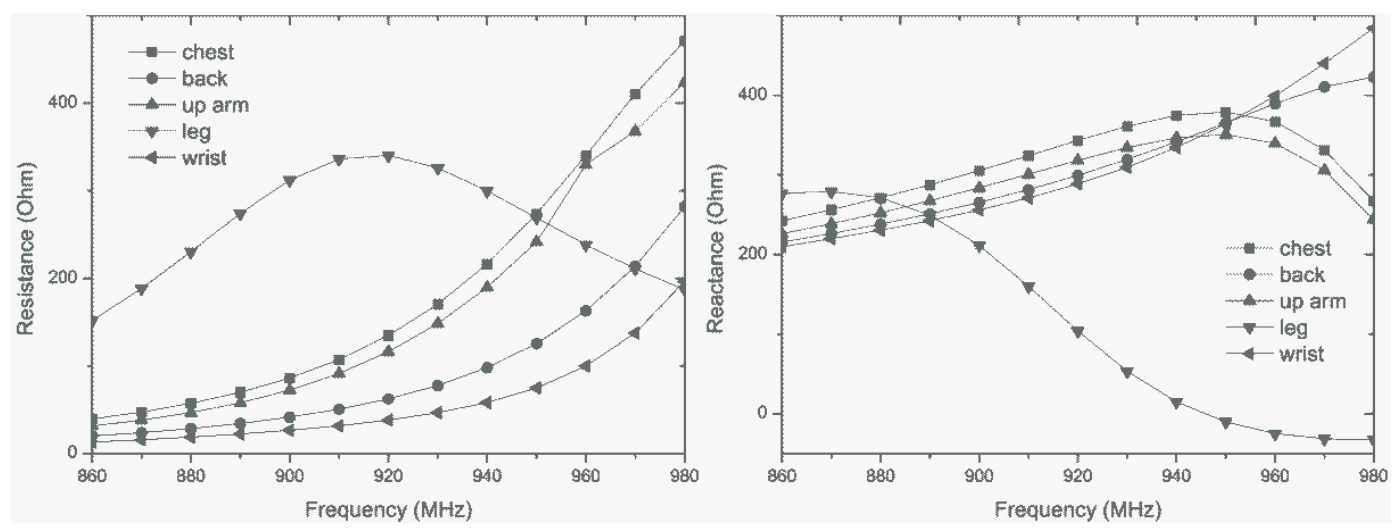

Fig.3. Simulated results of antenna impedance, resistance (A), reactance (B).

The gain of antenna indicates the degree of antenna's concentrated radiation by making use of input power. It is also an important factor to measure the performance of tag antenna, especially under different environments. In this study, 3D radiation patterns of simulation were created in CST to present the change of antenna performance with the tag attached on different positions of body. Radiation patterns of gain under five situations are reported in Fig.4.

From the values given by Fig.4(A-E), maximum calculated gains of tag antenna attached on different positions in descending order are chest $(-7.34 \mathrm{~dB})$, back $(-2.81 \mathrm{~dB})$, up arm $(-5.54 \mathrm{~dB})$, wrist $(-3.05 \mathrm{~dB})$, and thigh $(-6.74 \mathrm{~dB})$. It is found that the changes of gains differ with the tag attached on different positions of human body. The on-back tag has the maximum gain for not being bent and least effected by posture though affected by human tissue. Not the same with the on-back one, the tag antenna was bent when they attached on wrist, up arm and thigh, and the performance changed with more negative effects of bending, human posture and tissue. Therefore, antennas attached on these positions have smaller gain than the on-back one. Though the on-chest tag antenna is flat, it may be subject to some degrees of body's occlusion since the gesture has shielding effect on antenna. Thus, the on-chest tag has the worst performance in the five situations.

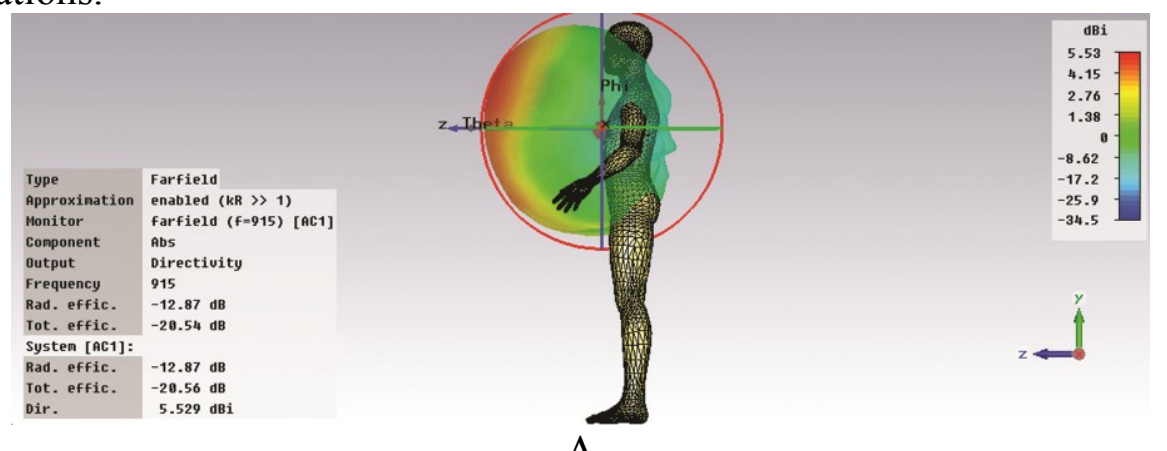

A

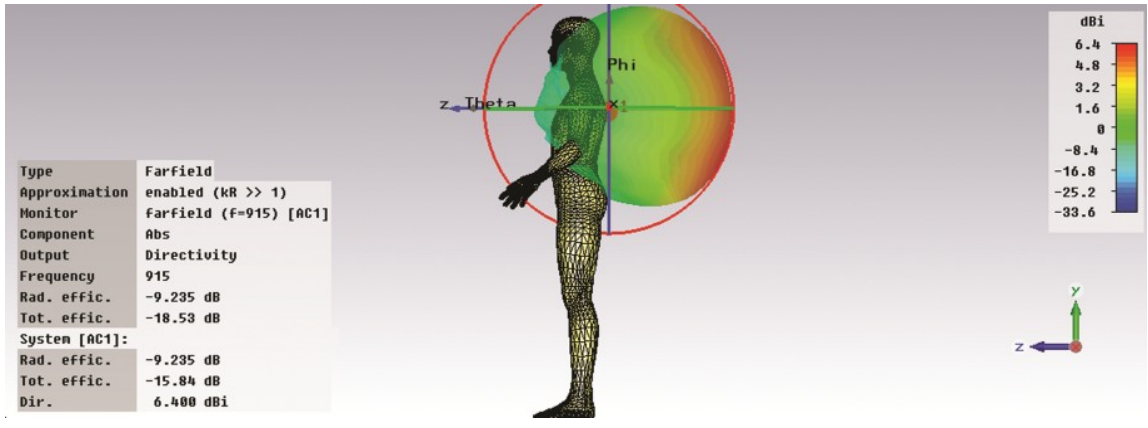




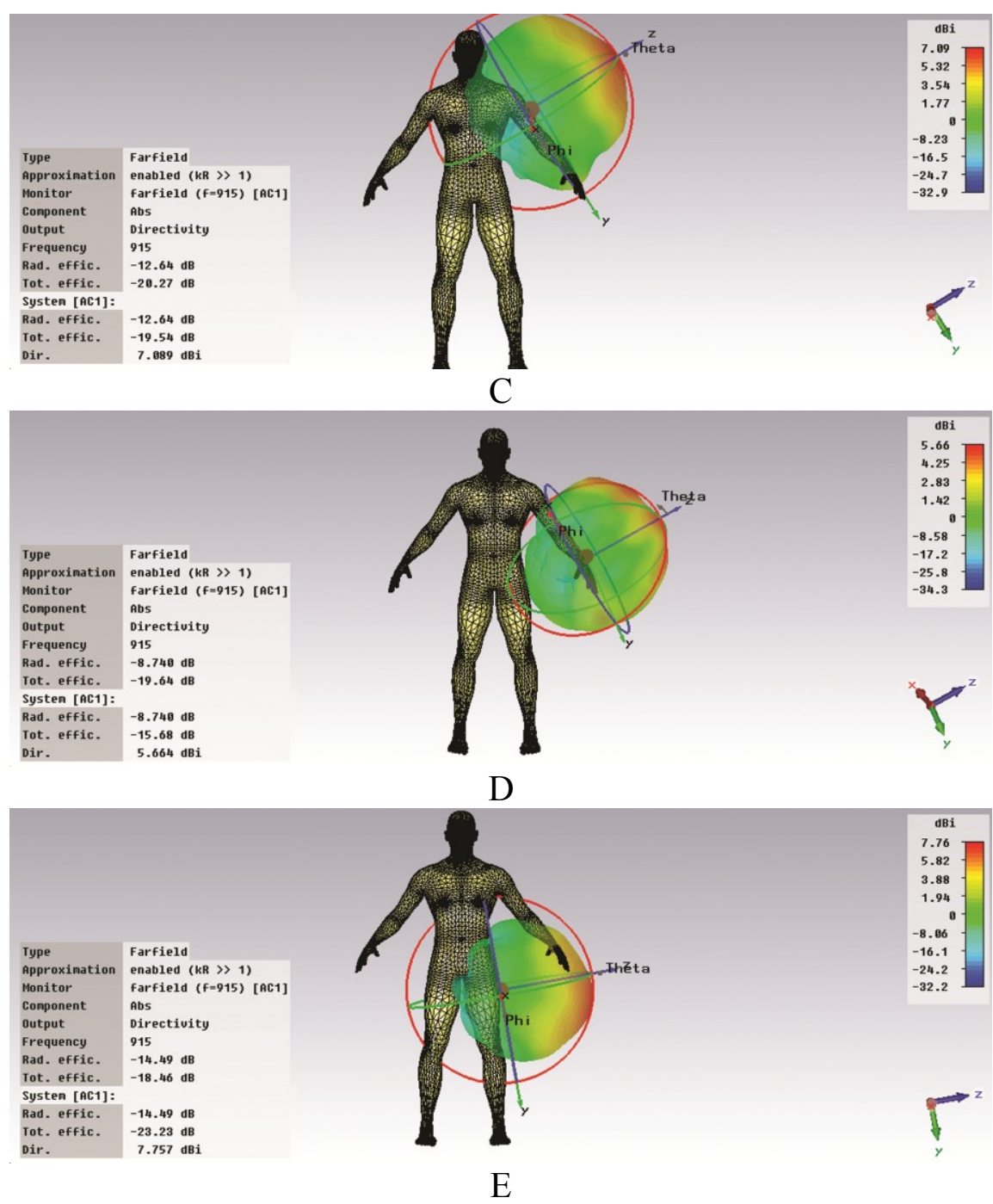

Fig.4. Simulated 3D radiation pattern results of tags attached on human body on five positions. Five positions of human are chest (Fig. A), back (Fig. B), wrist (Fig. C), up arm (Fig. D) and thigh (Fig. E).

\section{Results and Discussion}

A volunteer was selected to be the tested human for the same height with the model in simulation, and the volunteer with tag on his body can be seen in Fig. 5(A-E).The tag was attached as where they attached in simulation, and all settings of measurements were made to copy the ones in simulations. Five situations of tag attached on body were measured to get the maximum read distances of passive on-body tag.

In ideal situations, maximum read distance of a tag has connection with some parameters of tag antenna and the reader, such as the gain of tag antenna. Based on Friis formula, maximum read distance of a tag can be calculated by the value of antenna and other known parameters. Formula 1 presents the relationship between maximum read distance $(r)$ and some other parameters.

$$
r=\frac{\lambda}{4 \pi} \sqrt{\frac{P_{t} G_{t} G_{r} \tau P L F}{P_{t h}}}
$$

The value can be calculated with the formula given by 


$$
\tau=\frac{4 R_{c} R_{a}}{\left|z_{c}+z_{a}\right|^{2}} \quad 0<\tau<1
$$

Using two formulas given above, ideal read distances can be calculated with all parameters and results of simulation. Fig. 6 contains the results of ideal read distances and the measured maximum read distances.

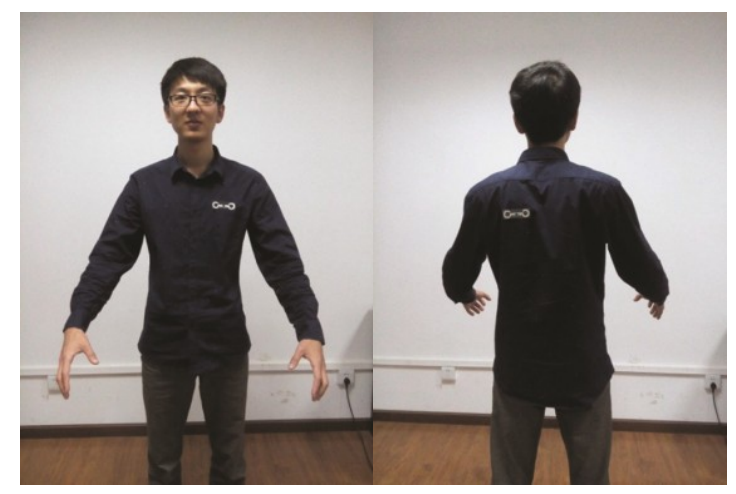

A

$\mathrm{B}$

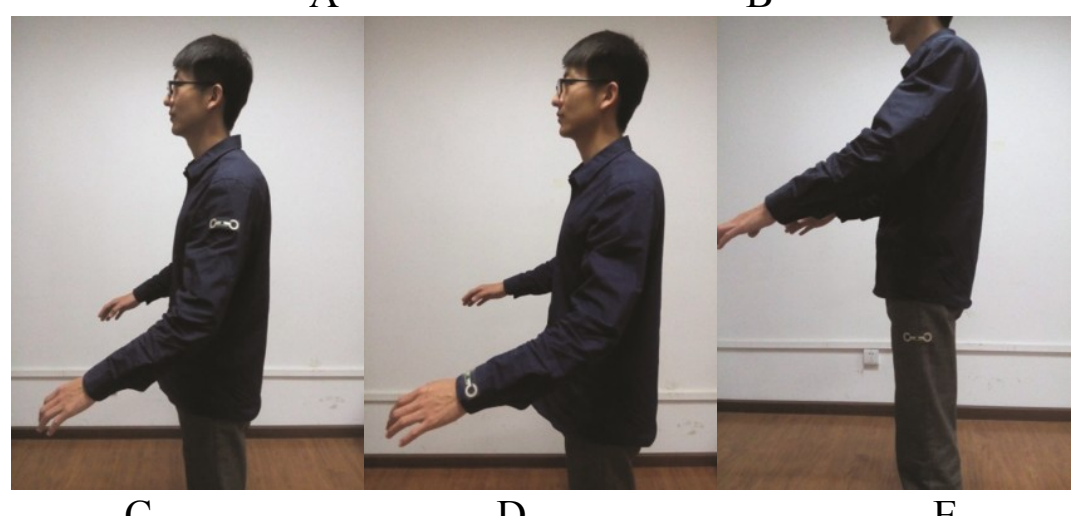

$\mathrm{C}$

$\mathrm{D}$

$\mathrm{E}$

Fig.5. Tag attached on different positions of the human body with the man keep one gesture. Tag attached on chest (Fig. 7A), back (Fig. 7B), up arm (Fig. 7C), wrist (Fig. 7D), thigh (Fig. 7E).

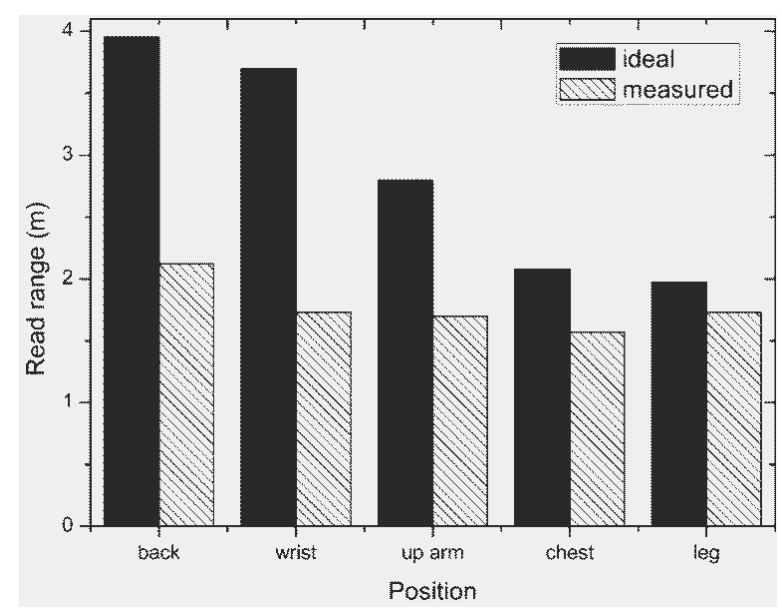

Fig.6. Ideal read distances and measured maximum read distances with tags attached on human.

As is shown in Fig.6, all the performance of tags was degenerated severely. Measurement result illustrate that the on-back tag has the best performance in five situations. The on-wrist and on-up arm tags also have better performance than the on-chest one, and it is consistent with the ideal read distances. It is obvious that the absorbing of body decreased the gains of tag antennas and the read distances shortened 
with gains decrease. To be rehabilitation training or security, printed tag on back is the best choice to get the maximum read distance.

\section{Conclusion}

In this paper, we have investigated the effects of five positions of human body on wearable tag antenna. Simulation was applied to research the tag antenna performance under ideal conditions, and printed tag was measured in reality. Both simulation and measurement have the same settings to research the reliability of tag under on-body environment. Calculated results of ideal read distances and measurement results demonstrated that different positions of human body influence antenna a lot. Back and wrist are suitable position to attach tag antennas and this provides convenience for future research.

\section{Acknowledgement}

This work is partly supported by the National Natural Science Foundation of China (No.51175394) and China Scholarship Council.

\section{References}

[1] Landt J. The history of RFID. IEEE Potentials, 2005.

[2] EPCglobal, Overview of the ultra high frequency (UHF) regulations worldwide, http://www.gs 1.org/epcglobal/implementation

[3] Want R. An introduction to RFID technology. IEEE Pervas Comput, 2006.

[4] Dobkin D. The RF in RFID - passive UHF RFID in practice. San Jose: WJ Communications, 2007.

[5] Karoliina Koski, Toni Björninen, Lauri Sydänheimo, Leena Ukkonen and Yahya Rahmat-Samii. A New Approach and Analysis of Modeling the Human Body in RFID -Enabled Body-CentricWireless Systems. Hindawi Publishing Corporation International Journal of Antennas and Propagation, 2014.

[6] J Virkki, T Björninen, T Kellomäki, S Merilampi, I Shafiq, L Ukkonen, L Sydänheimo, Y C Chan.Reliability of washable wearable screen printed UHF RFID tags. Microelectronics Reliability, April 2014.

[7] Zhi-Jun Tang, Yi-Gang He, Yan Wang. Broadband UHF RFID tag antenna with quasi-isotropic radiation performance. AEU - International Journal of Electronics and Communications, October 2011.

[8] T. Kellomäki, L. Ukkonen. Design approaches for body-worn RFID tags. International Symposium on Applied Sciences in Biomedical \& Communication Technologies, 2010.

[9] T Kellomäki, T Björninen, L Ukkonen, L. Sydänheimo. Shirt collar tag for wearable UHF RFID systems. European Conference on Antennas \& Propagation, 2010.

[10] Ansoft's High Frequency Structure Simulator HFSS. http://www.ansoft.com.11th version, 2008. 
[11]S Manzari, C Occhiuzzi,G Marrocco. Feasibility of Body-Centric Systems Using Passive Textile RFID Tags. Antennas \& Propagation Magazine IEEE, 2012.

[12] Scarpello M L, Kazani I, Hertleer C, Rogier H, Vande Ginste D.Stability and Efficiency of Screen-Printed Wearable and Washable Antennas. IEEE Antennas \& Wireless Propagation Letters, 2012 